[2] Benjamin DJ, Berger JO, Johannesson M, Nosek BA, Wagenmakers EJ, Berk R,et al. Redefine statistical significance. Nat Hum Behav. 2018;2:6-10.

[3] Wayant C, Scott J, Vassar M. Evaluation of lowering the p value threshold for statistical significance from .05 to .005 in previously published randomized clinical trials in major medical journals. JAMA. 2018;320:1813-15.

[4] Halsey, Lewis G., et al. The fickle P value generates irreproducible results. Nature methods. 2015;12:179-185.

Disclosure of Interests: None declared

DOI: 10.1136/annrheumdis-2020-eular.2608

\begin{tabular}{|l|l|}
\hline AB1223 & RHEUMATOID ARTHRITIS PATIENTS INCLUDED IN \\
GLUCOCORTICOID TRIALS MOSTLY RESEMBLE \\
THOSE SEEN IN OBSERVATIONAL COHORTS: A \\
SYSTEMATIC REVIEW AND META-ANALYSIS
\end{tabular}

A. Palmowski ${ }^{1}$, S. M. Nielsen ${ }^{2}$, T. Buttgereit ${ }^{1}$, Y. Palmowski ${ }^{1}$, M. Boers ${ }^{3}$, R. Christensen ${ }^{2}$, F. Buttgereit'. ${ }^{1}$ Charité - Universitätsmedizin Berlin, Berlin, Germany; ${ }^{2}$ Musculoskeletal Statistics Unit, The Parker Institute, Bispebjerg and Frederiksberg Hospital, University of Copenhagen and Research Unit of Rheumatology, Department of Clinical Research, University of Southern Denmark, Odense University Hospital, Frederiksberg and Odense, Denmark; ${ }^{3}$ Department of Epidemiology and Biostatistics, and Amsterdam Rheumatology and Immunology Center, Amsterdam UMC, Vrije Universiteit Amsterdam, Amsterdam, Netherlands

Background: Randomised controlled trials (RCTs) are considered the gold standard in clinical research. Their results, however, may not be generalizable to patients in routine care. ${ }^{1}$ Together with methotrexate, glucocorticoids (GCs) constitute the mainstay of therapy for many patients with rheumatoid arthritis (RA), but it is unclear whether trial evidence is actually generalizable to realworld patients.

Objectives: This review assesses to what extent RA patients participating in GC-RCTs differ from RA patients taking GCs in routine care.

Methods: This study was registered with PROSPERO (CRD42019134675). MEDLINE was searched for RCTs and, as comparators, cohort studies in RA evaluating systemic GC therapy. Cohorts were not allowed to exhibit apparent selection mechanisms concerning gender or age. Random-effects meta-analyses combined descriptive baseline characteristics that may modify the benefit-risk-ratio of various RA therapeutics. Meta-analyses were stratified by study type (RCT and CS). Stratified estimates were subsequently compared.

Results: 55 RCTs and ten cohort studies (21,657 participants overall) were included. Twelve characteristics (related to general demographics and disease activity) were reported frequently enough to allow for comparative analysis. Compared to cohorts, RCT participants were younger (-4.7 [-7.2 to -2.1$]$ years) and had somewhat higher erythrocyte sedimentation rates (12 [6 to 18$] \mathrm{mm} / \mathrm{h}$ ) (Table 1). In the other ten characteristics, estimates did not differ significantly. Numerically, cohort patients had more longstanding disease and slightly more favourable disease levels in core set variables. Comorbidities could not be assessed.

Table 1. Pooled estimates

\begin{tabular}{|c|c|c|c|c|c|c|c|}
\hline Outcome & RCT & $k$ & Cohort & $k$ & Contrast & $(95 \% \mathrm{Cl})$ & $p$ \\
\hline \multicolumn{8}{|l|}{ General demographics } \\
\hline Age (years) & 54.2 & 50 & 58.9 & 10 & -4.7 & $(-7.2$ to -2.1$)$ & $<0.001$ \\
\hline Female (proportion) & 0.70 & 52 & 0.73 & 10 & 0.89 & (0.68 to 1.16$)$ & 0.38 \\
\hline $\begin{array}{l}\text { Current or previous smokers } \\
\text { (proportion) }\end{array}$ & 0.59 & 3 & 0.51 & 2 & 1.38 & (0.61 to 3.14 ) & 0.44 \\
\hline $\operatorname{BMI}\left(\mathrm{kg} / \mathrm{m}^{2}\right)$ & 25.9 & 5 & 25.9 & 3 & 0.0 & $(-1.9$ to 1.9$)$ & 0.98 \\
\hline Disease duration (months) & 56.5 & 43 & 85.1 & 7 & -28.6 & ( -85.6 to 28.4$)$ & 0.33 \\
\hline \multicolumn{8}{|l|}{ Disease activity } \\
\hline $\operatorname{ESR}(\mathrm{mm} / \mathrm{h})$ & 40.1 & 31 & 28.2 & 3 & 11.8 & $(5.7$ to 18.0$)$ & $<0.001$ \\
\hline DAS & 5.3 & 24 & 4.9 & 5 & 0.4 & $(-0.1$ to 0.9$)$ & 0.12 \\
\hline RF+, (proportion) & 0.67 & 32 & 0.63 & 6 & 1.19 & (0.80 to 1.78 ) & 0.39 \\
\hline $\mathrm{ACPA}+$, (proportion) & 0.64 & 7 & 0.56 & 3 & 1.38 & (0.64 to 3.00$)$ & 0.41 \\
\hline $\mathrm{HAQ}$ & 1.3 & 31 & 1.1 & 4 & 0.2 & $(-0.1$ to 0.5$)$ & 0.15 \\
\hline Pain $(0-10)$ & 5.2 & 26 & 4.8 & 2 & 0.4 & $(-0.8$ to 1.6$)$ & 0.52 \\
\hline Patient global assessment $(0-10)$ & 5.2 & 17 & 4.9 & 3 & 0.3 & $(-0.9$ to 1.5$)$ & 0.58 \\
\hline
\end{tabular}

Conclusion: The results of our study suggest that evidence from RA GC-RCTs can be generalized to most patients in routine practice. We note that comorbidities - a frequent exclusion criterion for trial participation - could not be evaluated due to insufficient reporting. Our findings contrast with a similar study on RCTs investigating biologics in RA: There, trial participants were found to differ significantly in 4 out of 8 investigated baseline characteristics. ${ }^{2}$

References:

1]Palmowski $A$ et al. Applicability of trials in rheumatoid arthritis and osteoarthritis: A systematic review and meta-analysis of trial populations showing adequate proportion of women, but underrepresentation of elderly people. Semin Arthritis Rheum 2018 doi: 10.1016/j.semarthrit.2018.10.017 and

[2] Kilcher G et al. Rheumatoid arthritis patients treated in trial and real world settings: comparison of randomized trials with registries. Rheumatology (Oxford) 2017 doi: 10.1093/rheumatology/kex394

Acknowledgments: Part of the GLORIA project and trial, funded by the EU (Horizon 2020, Grant No 634886)

Disclosure of Interests: Andriko Palmowski: None declared, Sabrina Mai Nielsen: None declared, Thomas Buttgereit: None declared, Yannick Palmowski: None declared, Maarten Boers: None declared, Robin Christensen: None declared, Frank Buttgereit Grant/research support from: Amgen, BMS, Celgene, Generic Assays, GSK, Hexal, Horizon, Lilly, medac, Mundipharma, Novartis, Pfizer, Roche, and Sanofi.

DOI: 10.1136/annrheumdis-2020-eular.732

\section{AB1224 MAJOR DEPRESSIVE DISORDER AMONG CYPRIOT PATIENTS WITH SYSTEMIC LUPUS ERYTHEMATOSUS AND CORRELATION WITH CLINICAL CHARACTERISTICS AND DISEASE-SPECIFIC HEALTH- RELATED QUALITY OF LIFE}

K. Parperis ${ }^{1,2}$, S. Psarelis ${ }^{3}$, S. Symeonidou ${ }^{3}$, M. Michaelides ${ }^{4}$, D. Nikiforou ${ }^{5}$, E. Antoniade ${ }^{1}$, B. Bhattarai ${ }^{2,6}{ }^{1}{ }^{1}$ University of Cyprus Medical School, Nicosia, Cyprus; ${ }^{2}$ The University of Arizona College of Medicine, Phoenix, United States of America; ${ }^{3}$ Nicosia General Hospital, Strovolos, Cyprus; ${ }^{4}$ Ygia Polyclinic Private Hospital, Limassol, Cyprus; ${ }^{5}$ Limassol General Hospital, Kato Polemidia, Cyprus; ${ }^{6}$ Valleywise Health Medical Center, Phoenix, United States of America

Background: Major depressive disorder (MDD) is a common comorbidity in patients with chronic rheumatic conditions, and patients with systemic lupus erythematous (SLE) have an increased risk of developing depression. The coexistence of SLE and MDD might lead to impaired health-related quality of life (HRQOL). Gaining a deeper understanding of the factors associated with MDD in SLE will allow us to develop strategies in order to prevent depression and achieve an early diagnosis and management.

Objectives: The aim of this study is to determine the contributing factors associated with MDD in Cypriot SLE patients and examine the correlation between disease-specific HRQOL domains and depression.

Methods: We conducted a cross-sectional study of SLE patients who fulfilled the SLICC-2012 criteria. The patients were recruited from August 2019 to January 2020. Baseline demographic data, clinical features, and therapeutic regimens were captured. Depression was assessed by the patient health questionnaire (PHQ)-9, a validated tool to screen and diagnosed the condition. Scores above $\geq 10$ are indicating MDD. HRQOL was evaluated by a disease-specific validated questionnaire, LupusQoL-Greek. LupusQol scores ranged between 0-100, with higher scores reflecting better QoL. The self-rated health status was examined, and the response was separate to poor/fair vs excellent/good. Demographic data, LupusQoL domains, clinical and other features of the SLE patients were described and compared between MDD (PHQ-9 $\geq 10$ ) and non-MDD (PHQ$9<10)$ groups using Wilcoxon ranksum tests for continuous variables and chisquare tests for categorical variables.

Results: A total of 88 SLE patients were included in the study, with a mean age of $48.6(19-80), 71(80 \%)$ were women, and mean disease duration of 13.2 years (0-44). Compared to the non-MDD group, patients with MDD ( $n=32,36 \%)$ were significantly older (mean age 53.5 vs $46.9 ; p=0.03$ ) and more likely to have the following SLE manifestations: mucocutaneous, vascular, pulmonary and musculoskeletal involvement. Furthermore, patients with MDD were less likely to be clinical quiescent $(34.3 \%)$ compared with the non-MDD group $(57.1 \%)$ $(P<0.05)$. Self-rated health described as poor/fair was markedly associated with MDD $(p<0.001)$. The LupusQoL domains' scores were notably lower in patients with MDD (PHQ $\geq 10)$, with a statistically significant reduction in all LupusQoL domains, indicating that depression was associated with worse HRQOL (Table 1).

Table 1. Differences in 8 LupusQoL domain scores among SLE patients with and without major MDD (PHQ-9 $\geq 10$ )

\begin{tabular}{lccc}
\hline $\begin{array}{l}\text { LupusQoL Domains } \\
\text { (0-100) }\end{array}$ & $\begin{array}{c}\text { PHQ-9 }<10 \\
\text { (non-MDD) }\end{array}$ & $\begin{array}{c}\text { PHQ-9 } \geq 10 \\
\text { (MDD) }\end{array}$ & P-value \\
\hline Physical Health & $72.87(81.2,9.3-100)$ & $40.01(32.75,0-90.6)$ & $<0.0001$ \\
Pain & $75.27(75,16.6-100)$ & $43.2(41.6,0-100)$ & $<0.0001$ \\
Planning & $81.08(91.6,0-100)$ & $44.51(50,0-100)$ & $<0.0001$ \\
Intimate relationship & $68.38(75,0-100)$ & $46.25(50,0-100)$ & 0.0382 \\
Burden to others & $79.73(83.3,16-100)$ & $51.97(50,0-100)$ & 0.0003 \\
Emotional health & $81.76(85.4,25-100)$ & $50.14(50,0-91.6)$ & $<0.0001$ \\
Body image & $84.09(90.8,5-100)$ & $58.45(66.6,0-100)$ & 0.0004 \\
Fatigue & $72.08(75,18.7-100)$ & $41.2(40.6,6.2-81.2)$ & $<0.0001$ \\
\hline
\end{tabular}


Conclusion: To our knowledge, this is the first study demonstrating significantly lower scores among MDD relative non-MDD (based on PHQ-9) patients across all the 8 domains of LupusQoL; therefore, it adds to the current evidence that depression significantly impacts the quality of life of SLE. Predictors of MDD include older age, and involvement of the vascular, mucocutaneous, pulmonary, and musculoskeletal system. Additionally,a poor or fair description of self-rated health was a predictor of depression.

Disclosure of Interests: None declared

DOI: 10.1136/annrheumdis-2020-eular.946

\section{AB1225 LIPID PROFILE COMPARISON IN RHEUMATOID ARTHRITIS AND PSORIATIC ARTHRITIS: A CASE- CONTROL STUDY}

D. Á. Galarza-Delgado ${ }^{1}$, J. R. Azpiri-López ${ }^{2}$, I. J. Colunga-Pedraza ${ }^{1}$, A. Pérez Villar $^{1}$, I. C. Zárate Salinas ${ }^{2}$, P. F. Frausto Lerma ${ }^{1}$, M. A. Reyes Soto ${ }^{1}$, D. E. Flores Alvarado ${ }^{1}$, O. Ilizaliturri Guerra ${ }^{1}{ }^{1}$ Hospital Universitario Dr. José Eleuterio González, Rheumatology Department, Monterrey, Mexico; ${ }^{2}$ Hospital Universitario Dr. José Eleuterio González, Cardiology Department, Monterrey, Mexico

Background: Rheumatoid arthritis (RA) and psoriasic arthritis (PsA) are autoimmune diseases, in both diseases it has been described that the main cause of morbidity and mortality is cardiovascular (CV) disease. Dyslipidemia is the most recognized $\mathrm{CV}$ risk factor. An association is recognized between the concentrations of total cholesterol (TC), high-density lipoprotein cholesterol (HDL-C), low-density lipoprotein cholesterol (LDL-C), total triglycerides (TG), atherogenic index (AI) and the risk of myocardial infarction (MI), stroke and fatal cardiovascular disease (CVD). The relationship between serum lipid levels and CVD risk is potentially paradoxical in RA but this relationship has not been clarified in PsA. Objectives: To compare lipid profile between groups with RA, PsA and controls. Methods: A cross-sectional observational study was designed, which included 95 patients between 45-75 years who fulfilled the CASPAR classification criteria for PsA. 95 patients between 45-75 years who fulfilled the ACR / EULAR 2010 classification criteria for RA and 95 age-matched controls. Concentrations of CT, HDL-C, LDL-C, TG and atherogenic index were compared between the groups. Clinical measures were compared using one-way ANOVA or Kruskall-Wallis tests. Post-hoc analysis was performed with Bonferroni's correction. A $p \leq 0.05$ was considered statistically significant. The data was analyzed using the SPSS version 25 software package.

Results: In our study, no significant difference in LDL-C was found between RA and PsA, however post-hoc analysis was performed where we found higher LDL-C levels among RA patients compared with controls ( $p$ 0.025). RA patients had higher HDL-C than PsA patients ( $p$ 0.006) but PsA had a higher HDL-C than controls $(p$ 0.007). TC/HDL-C was higher in PsA than RA and controls $(p 0.050)$. PsA patients were the group with the lowest HDL-C levels $(p 0.007)$. In contrast RA were the groups with the highest HDL-C levels ( $p$ 0.007). (Table 1).

Table 1. Clinical parameters.

\begin{tabular}{lcccc}
\hline PARAMETER & RA & PSA & Controls & $p$ \\
\hline TC $^{\star}$ & $176.6 \pm 37.2$ & $176.3 \pm 35.9$ & $186.34 \pm 33.172$ & 0.089 \\
TG $^{* *}$ & $132.7(102.0-187.3)$ & $131.0(97.2-189.2)$ & $118.35(88.2-162.25)$ & 0.245 \\
HDL-C** & $50.7(42.1-62.6)$ & $46.7(37.4-53.9)$ & $51.7(41.3-60)$ & $\mathbf{0 . 0 0 7}$ \\
LDL-C $^{*}$ & $94.36 \pm 21.70$ & $97.71 \pm 30.12$ & $105.32 \pm 31.35$ & $\mathbf{0 . 0 2 5}$ \\
TC/HDL-C** $^{*}$ & $3.41(2.81-4.08)$ & $3.74(3.17-4.47)$ & $3.49(2.99-4.52)$ & $\mathbf{0 . 0 5 0}$ \\
\hline
\end{tabular}

*Data are reported in mean $\pm \mathrm{SD}$

${ }^{* *}$ Data is reported in median (IQR)

Conclusion: Patients with inflammatory joint diseases have more adverse lipid profiles than controls.

References:

[1] Pietrzak, A., Chabros, P., Grywalska, E., Kiciński, P., Pietrzak-Franciszkiewicz, K., Krasowska, D., \& Kandzierski, G. (2019). Serum lipid metabolism in psoriasis and psoriatic arthritis-an update. Archives of medical science: AMS, 15(2), 369

Disclosure of Interests: None declared

DOI: 10.1136/annrheumdis-2020-eular.5805

$\mathrm{AB} 1226$

\section{PREDICTING CARDIOVASCULAR EVENTS IN} PATIENTS WITH SPONDYLOARTHRITIS AND PSORIATIC ARTHRITIS: THREE RISK ALGORITHMS

A. S. Pinto ${ }^{1}$, F. Cunha Santos ${ }^{1}$, S. P. Dinis ${ }^{1}$, J. F. Ferreira ${ }^{1,2}$, C. Vaz $^{1,2} .{ }^{1}$ Local Health Unit of Guarda, Guarda, Portugal; ${ }^{2}$ Beira Interior University, Covilhã, Portugal
Background: Inflammatory joint disorders have an increased cardiovascular (CV) risk when compared with general population. In 2009, the EULAR task force advocated the use of a 1.5 multiplication factor for these risk prediction models in Rheumatoid Arthritis patients with specific characteristics. The SCORE was not modified in patients with Psoriatic Arthritis (PsA) and Spondyloarthritis (SpA) in accordance with the present EULAR recommendations.

Objectives: To assess the accuracy of several CV risk algorithms to predict an event and determine its sensibility and specificity.

Methods: A retrospective analysis of PsA and SpA patients, followed in our department in Local Health Unit of Guarda during one year (2019) was done. We determined CV risk profile of our patients using the following data: gender, age, smoking status, blood pressure, lipid values and diabetes mellitus status. These variables were used to calculate risk prediction algorithms such as Framingham, the American College of Cardiology/American Heart Association (ACC/AHA) risk score and the Systematic Coronary Risk Evaluation (SCORE). Discriminatory ability for CV risk prediction was evaluated by the area under the ROC curves. Sensibility and specificity were calculated for low-to-intermediate and intermediate-to-high risk cut-offs. Cut-off values that mark the high risk were defined in $5 \%$ for SCORE, 20\% for Framingham and ACC/AHA.

Results: 112 caucasic patients were included, $61 \%$ female with a mean age of $52.15 \pm 14.18$ years and mean BMI of $27.11 \pm 4.81 \mathrm{~kg} / \mathrm{m}^{2} .7$ patients weren't eligible to apply these cardiovascular scores. 8 patients were identified with non-fatal cardiovascular events ( 2 cases of stroke, 5 cases of myocardial infarction and 1 case of thrombophlebitis). 69 patients diagnosed with $\mathrm{SpA}$ and 43 with PsA five CV events identified in SpA patients and three in PsA patients. Area unde the ROC in SpA were 0.729 (95\% Cl 0.461 to 0.996) for SCORE, 0.839 (95\% C 0.735 to 0.943 ) for Framingham and 0.804 (95\% Cl 0.683 to 0.925$)$ for ACC/AHA Area under the ROC in PsA patients were $0.603(95 \% \mathrm{Cl} 0.327$ to 0.879$)$ for SCORE, 0.660 (95\% Cl 0.326 to 0.995$)$ for Framingham and $0.804(95 \% \mathrm{Cl} 0683$ to 0.925$)$ for ACC/AHA. Sensibility and specificity were discriminated in table 1.

Table 1. Sensibility, specificity, positive predictive value (PPV) and negative predictive value (NPV) of CV risk algorithms

\begin{tabular}{lcccc}
\hline & Sensibility (\%) & Specificity (\%) & PPV (\%) & NPV (\%) \\
\hline SCORE > 1\% & & & & \\
SpA & 75 & 63 & 18 & 96 \\
PsA & 100 & 41 & 5 & 100 \\
SCORE > 5\% & & & & \\
SpA & NA & NA & NA & NA \\
PsA & 0 & 93 & 0 & 97 \\
Framingham >10\% & & & & \\
SpA & 83 & 78 & 28 & 98 \\
PsA & 50 & 51 & 5 & 95 \\
Framingham $>$ 20\% & & 86 & 20 & 93 \\
SpA & 33 & 69 & 8 & 96 \\
PsA & 50 & 77 & 29 & 94 \\
ACC/AHA $>$ 5\% & & 44 & 9 & 100 \\
SpA & 67 & & & \\
PsA & 100 & 86 & 14 & 88 \\
ACC/AHA $>\mathbf{2 0} \%$ & 17 & 83 & 14 & 97 \\
SpA & 50 & &
\end{tabular}

Conclusion: A good discrimination between patients with or without CV events has been demonstrated by area under the ROC curve, particularly to SpA patients. In PsA, the sample was smaller, which represents a limitation in this study. SCORE $>5 \%$ did not identify CV events, therefore sensibility couldn't be calculated. Overall, the algorithms studied presented a low sensibility, underestimating CV risk. This could be explained since disease-related factors are not reflected in these scores. In our opinion, better algorithms are needed to correctly assess cardiovascular risk algorithms for $\mathrm{SpA}$ and $\mathrm{PsA}$, since the majority of the events occur in patients with low-intermediate risk.

References:

[1] Agca R, Heslinga SC, Rollefstad S, et al. EULAR recommendations for cardiovascular disease risk management in patients with rheumatoid arthritis and other forms of inflammatory joint disorders: 2015/2016 update, Ann Rheum Dis 2017;76: 17-28.

Disclosure of Interests: None declared

DOI: 10.1136/annrheumdis-2020-eular.3972

\section{AB1227 PREVALENCE OF RHEUMATIC MUSCULOSKELETAL DISORDERS IN A RURAL AREA OF UPPER EGYPT: WHO-ILAR COPCORD BASED COMMUNITY STUDY}

S. Rashad ${ }^{1}$, E. Abda ${ }^{2}$, Z. Selim ${ }^{3}$, S. Hussein ${ }^{2}$, T. Metwally ${ }^{4}$, D. Fouad ${ }^{5}{ }^{1}$ Suez University, Rheumatology \& Rehabilitation, Suez, Egypt; ${ }^{2}$ Assiut University, Rheumatology \& Rehabilitation, Assiut, Egypt; ${ }^{3}$ Assiut, Rheumatology \& Rehabilitation, Assiut, Egypt; ${ }^{4}$ Suez University, Family Medicine, Suez, Egypt; ${ }^{5}$ South Egypt Cancer Institute, Radiology, Assiut, Egypt 